\title{
Kestabilan Global Endemik Model Epidemi SEIR
}

\author{
Global Stability Endemic of SEIR Epidemic Model \\ Roni Tri Putra ${ }^{1)}$, Sukatik $^{2)}$, Sri Nita $^{3)}$ \& Yandraini Yunida ${ }^{4)}$ \\ 1),2) Jurusan Teknik Sipil, Politeknik Negeri Padang, Kampus Unand Limau Manis Padang, Telp. \\ 0751-72590 Fax.0751-72576 Email: putra_tryronny@yahoo.co.id \\ dan atik_wiryosentono@yahoo.co.id \\ 3),4) Jurusan Teknik Elektro, Politeknik Negeri Padang, Kampus Unand Limau Manis Padang, Telp. \\ 0751-72590 Fax. 0751-72576 Email : srinita0610@ gmail.com \\ dan yandraini_ec@yahoo.co.id
}

\begin{abstract}
In this paper, it will be studied global stability endemic of equilibrium points of a SEIR model with infectious force in latent, infected and immune period. From the model it will be found investigated the existence and its stability of points its equilibrium. The global stability of equilibrium points is depending on the value of the basic reproduction number $\left(R_{0}\right)$. If $R_{0}>1$ there is a unique endemic equilibrium which is globally asymptotically stable.
\end{abstract}

Keywords : a basic reproduction number, stability, equilibrium points

\section{PENDAHULUAN}

Penerapan model matematika dan teknik matematika untuk mendalami masalah biosciences dipelajari dalam mathematical biosciences. Salah satu cabang mathematical biosciences adalah mathematical epidemiology, yang mempelajari tentang penyebaran dan pengendalian penyakit. Mempelajari model epidemi yang didalamnya termasuk penyakit penyebab kematian pada suatu populasi total yang berubah merupakan hal penting dalam mathematical epidemiology.

Epidemiology merupakan suatu cabang ilmu yang mempelajari bagaimana terjadi epidemic dalam suatu populasi makhluk hidup. Dengan menggunakan pemodelan matematika yang didasarkan pada asumsiasumsi tertentu, diharapkan dari model yang disusun dapat menjelaskan fenomena dan mengambil tindakan apa yang harus dilakukan jika terjadi epidemi.

Ada berbagai model matematika epidemi yang dikenal berdasarkan sifat atau ciri - ciri penyakitnya. Misalnya $S, E, I$ dan $R$ berturut - turut menunjukkan Susceptible (kelas populasi yang rentan),
Exposed

Infectious

(kelas populasi yang laten), terinfeksi) dan Recovered (kelas populasi yang sembuh).

Dengan berbagai asumsi, dikenal berbagai model epidemi, diantaranya SIR, SIRS, SEIR, SEIRS dan SEIS. Dalam model SIR, individu yang sembuh mempunyai kekebalan sehingga tidak lagi menjadi rentan, sedangkan untuk model SIRS individu yang sudah sembuh tidak memiliki kekebalan terhadap penyakit tersebut sehingga dapat menjadi rentan lagi. Dalam model SEIR, SEIRS dan SEIS individu yang rentan melalui masa laten setelah terinfeksi sebelum menjadi terjangkit.

Beberapa penyakit seperti cacar air (measles), gondong (mumps), tubercoluses, Human Immunodeficiency Virus (HIV) mempunyai periode laten (laten period). Periode laten adalah selang waktu dimana suatu individu terinfeksi sampai munculnya penyakit. Adanya periode laten ini menjadi alas an pembentukan model epidemi SEIR, yakni munculnya kelas exposed. Dalam tulisan ini hanya akan dibahas model epidemi SEIR. 
Dari berbagai literatur belum banyak yang mengkaji model matematika epidemi SEIR secara sistematis. Sejalan dengan masalah yang akan dibahas, penelitian ini mempunyai tujuan sebagai berikut :

1. Membentuk model matematika epidemi SEIR pada populasi manusia

2. Menentukan titik - titik ekuilibrium model tersebut

3. Menyelidiki kestabilan titik - titik ekuilibrium model tersebut

4. Mengaplikasikan kriteria kestabilan matriks pada model epidemi SEIR.

Hasil penelitian ini diharapkan dapat :

1. Secara umum diharapkan dapat memberikan manfaat dan sumbangan terhadap ilmu pengetahuan, serta untuk menambah wawasan khususnya dalam bidang matematika terapan.

2. Secara khusus diharapkan dapat memberikan gambaran tentang model epidemi SEIR dengan kemampuan infeksi pada kelas laten, infeksi dan sembuh.

Kermack dan McKendrick (1927) telah memperkenalkan model epidemi. Dalam penelitian ini dipertimbangkan 4 kelas populasi yaitu $S$ (rentan), $E$ (laten), $I$ (infeksi) dan $R$ (sembuh). Anderson (1979) mempertimbangkan masalah ini dalam suatu tikus - tikus percobaan. Pearl dan Reed (1920) memperkenalkan model pertumbuhan logistik. Perko (1991) menjelaskan pengertian titik ekuilibrium pada sistem persamaan diferensial dan menjelaskan definisi nilai eigen dan vektor eigen. Arrowsmith (1992) menjelaskan transformasi dengan matriks Jordan dan sistem persamaan diferensial linear dengan matriks berukuran $2 \times 2$.

Salah satu masalah klasik yang sering dihadapi dalam matematika epidemi adalah menganalisis kestabilan global titik ekuilibrium. Kestabilan itu sendiri ada yang bersifat lokal dan ada yang bersifat global. Kestabilan lokal mudah ditentukan dengan pendekatan linier, sedangkan kestabilan global cukup sulit ditentukan.

\section{METODOLOGI}

Metode penelitian dalam tulisan ini adalah dengan mempelajari jurnal - jurnal dan buku - buku yang berhubungan dengan penyakit epidemi, khususnya model SEIR. Oleh karenanya materi yang diteliti sebagian besar bersumber dari hasil karya ilmiah para pakar matematika dalam bentuk buku. Prosedur penelitian diawali dengan pemisalan, menentukan asumsi dan mendefinisikan parameter yang digunakan pada model. Setelah itu dibentuk model matematika epidemi SEIR tersebut.

Selanjutnya, menentukan titik - titik ekuilibrium model tersebut dengan menggunakan definisi titik ekuilibrium suatu sistem persamaan diferensial. Setelah menentukan titik - titik ekuilibrium model tersebut, langkah selanjutnya menyelidiki sifat kestabilan lokal titik - titik ekuilibrium model tersebut. Untuk menyelidiki kestabilan lokal dilakukan linearisasi pada sistem dengan menentukan matriks Jacobian di titik ekuilibrium. Selanjutnya menentukan nilai eigen dari matriks Jacobian tersebut dengan menggunakan definisi polinomial karakteristik suatu matriks. Salah satu alternatif menentukan nilai eigen dari polinomial karakteristik suatu matriks digunakan juga Teorema Routh - Hurwitz.

Selain itu, sifat kestabilan lokal titik ekuilibrium pada sistem dapat ditentukan dengan fungsi Lyapunov dan menggunakan teorema himpunan invarian. Selanjutnya, dengan menggunakan teorema himpunan invarian, jika dapat diperluas sehingga berlaku untuk seluruh domain sistem maka berlaku sifat kestabilan global.

\section{HASIL DAN PEMBAHASAN}

Model SEIR diterapkan pada penyakit yang memiliki masa inkubasi cukup lama. Pada umumnya selama masa laten tersebut individu tidak bisa menularkan penyakit, tetapi pada beberapa 
penyakit seperti malaria, individu yang berada dalam masa laten, infeksi dan sembuh mempunyai kemungkinan untuk menularkan penyakit. Pada model SEIR populasi dibagi menjadi 4 kelas (subpopulasi) yaitu

a. Kelas $S$ (Susceptible) menyatakan kelas individu yang rentan

b. Kelas $E$ (Exposed) menyatakan kelas individu yang telah terinfeksi tapi belum sakit (laten)

c. Kelas I (Infectious) menyatakan kelas individu yang sudah terjangkit penyakit

d. Kelas $R$ (Recovered) menyatakan kelas individu yang telah sembuh dari penyakit.

Pada pemodelan yang akan dibentuk, digunakan asumsi - asumsi sebagai berikut

a. Populasi terbuka

b. Individu yang berada dalam kelas laten, infeksi dan sembuh mempunyai kemungkinan untuk menularkan penyakit

c. Penyakit menyebabkan kematian (fatal)

d. Masa inkubasi cukup lama.

Definisi dari parameter - parameter yang digunakan pada model adalah $A$ menyatakan konstanta rekruitmen dari $S$ $\beta_{10}$ menyatakan laju dari kontak efektif pada periode laten

$\beta_{20}$ menyatakan laju dari kontak efektif pada periode infeksi

$\beta_{30}$ menyatakan laju dari kontak efektif pada periode sembuh

$\mu$ menyatakan laju kematian alami

$\alpha_{10}$ menyatakan laju kematian karena penyakit pada kelas $E$

$\alpha_{20}$ menyatakan laju kematian karena penyakit pada kelas $I$

$\gamma_{0}$ menyatakan laju dari kelas $E$ ke kelas $I$

$k_{0}$ menyatakan laju kesembuhan.

Formulasi modelnya adalah :

$\frac{d S}{d t}=A-\beta_{10} S E-\beta_{20} S I-\beta_{30} S R-\mu S$

$$
\begin{aligned}
& \frac{d E}{d t}=\beta_{10} S E+\beta_{20} S I+\beta_{30} S R-\gamma_{0} E- \\
& \left(\mu+\alpha_{10}\right) E
\end{aligned}
$$

$\frac{d I}{d t}=\gamma_{0} E-k_{0} I-\left(\mu+\alpha_{20}\right) I$

$\frac{d R}{d t}=k_{0} I-\mu R$.

Diberikan $N(t)$ menyatakan ukuran populasi pada saat $t$, maka

$$
N(t)=S(t)+E(t)+I(t)+R(t) .
$$

Untuk menyederhanakan Sistem (1) s.d (4) dimisalkan $\mu d t=d \tau$ atau $\tau=\mu t$, maka Sistem (1) s.d (4) menjadi :

$\frac{d S}{d \tau}=\frac{A}{\mu}-\beta_{1} S E-\beta_{2} S I-\beta_{3} S R-S$

$\frac{d E}{d \tau}=\beta_{1} S E+\beta_{2} S I+\beta_{3} S R-(1+\gamma+$

$\left.\alpha_{1}\right) E$

$\frac{d I}{d \tau}=\gamma E-\left(1+k+\alpha_{2}\right) I$

$\frac{d R}{d \tau}=k I-R_{y}$

dengan

$\beta_{1}=\frac{\beta_{10}}{\mu}, \beta_{2}=\frac{\beta_{20}}{\mu}, \beta_{3}=\frac{\beta_{g 0}}{\mu}, \gamma=\frac{\gamma_{0}}{\mu}, \alpha_{1}=$ $\frac{\alpha_{10}}{\mu}, \alpha_{2}=\frac{\alpha_{20}}{\mu}, k=\frac{k_{0}}{\mu}$.

Jika $S=N-E-I-R$ disubstitusikan ke dalam Sistem (5) s.d (8) diperoleh

$\frac{d E}{d \tau}=\left(\beta_{1} E+\beta_{2} I+\beta_{3} R\right)(N-E-I-R)-$ $\delta E$

$\frac{d I}{d \tau}=\gamma E-\omega I$

$\frac{d R}{d \tau}=k I-R$ 
$\frac{d N}{d \tau}=\frac{A}{\mu}-N-\alpha_{1} E-\alpha_{2} I$,

dengan

$$
\delta=1+\gamma+\alpha_{1}
$$

$\omega=1+k+\alpha_{2}$.

\section{Titik Ekuilibrium}

Titik ekuilibrium Sistem (5) s.d (8) diperoleh dengan menjadikan ruas kanan masing - masing persamaan sama dengan nol, dan diperoleh :

$S=\frac{A\left(\alpha_{1} \omega+\alpha_{2} \gamma\right)}{\left(\beta_{1} \omega+\beta_{2} \gamma+\beta_{\mathrm{g}} k \gamma\right)(A-\mu N)+\mu\left(\alpha_{1} \omega+\alpha_{2} \gamma\right)}$

$E=\frac{\omega[A-\mu N]}{\mu\left(\omega \alpha_{1}+\gamma \alpha_{2}\right)}$

$I=\frac{\gamma[A-\mu N]}{\mu\left(\alpha_{1} \omega+\alpha_{2} \gamma\right)}$

$R=\frac{k \gamma[A-\mu N]}{\mu\left(\alpha_{1} \omega+\alpha_{2} \gamma\right)}$

Berikut diberikan teorema sifat kestabilan titik ekuilibrium endemik pada Sistem (9) s.d (12).

Teorema 1. Jika $R_{0}>1$ maka

a. Titik ekuilibrium bebas penyakit $P_{0}\left(0,0,0, \frac{A}{\mu}\right)$ pada Sistem (9) s.d (12) tidak stabil,

b. Titik ekuilibrium endemik $P^{*}=\left(E^{*}{ }_{,} I^{*}, R^{*}, N^{*}\right)$ pada Sistem

(9) s.d (12) merupakan stabil asimtotik lokal.

\section{Bukti}

a. Matriks Jacobian dari Sistem (9) s.d

(12) pada titik ekuilibrium $P^{*}=\left(E^{*}, I^{*}, R^{*}, N^{*}\right) \quad$ adalah

If $\left(P^{*}\right)$, dengan

$$
n=\beta_{1} E^{*}+\beta_{2} I^{*}+\beta_{3} R^{*} .
$$

Perhatikan bahwa

$$
\beta_{1}\left(N^{*}-E^{*}-I^{*}-R^{*}\right)=\frac{\beta_{1} \delta E^{*}}{\beta_{1} E^{*}+\beta_{2} I^{*}+\beta_{\mathrm{g}} R^{*}}
$$

$$
\Leftrightarrow \beta_{1}\left(N^{*}-E^{*}-I^{*}-R^{*}\right)=
$$$$
\frac{\frac{\beta_{1} \delta \omega(A-\mu N)}{\mu\left(a_{1} \omega+a_{2} \gamma\right)}}{\frac{\left(\omega \beta_{1}+\gamma \beta_{2}+k \gamma \beta_{8}\right)(A-\mu N)}{\mu\left(a_{1} \omega+a_{2} \gamma\right)}}
$$

$\Leftrightarrow \beta_{1}\left(N^{*}-E^{*}-I^{*}-R^{*}\right)=$

$\frac{\beta_{1} \delta \omega}{\left(\omega \beta_{1}+\gamma \beta_{2}+k y \beta_{\Omega}\right)^{*}}$

Dengan cara yang sama diperoleh

$$
\begin{aligned}
& \beta_{2}\left(N^{*}-E^{*}-I^{*}-R^{*}\right)=\frac{\beta_{2} \delta \omega}{\left(\omega \beta_{1}+\gamma \beta_{2}+k \gamma \beta_{2}\right)} \\
& \beta_{3}\left(N^{*}-E^{*}-I^{*}-R^{*}\right)=\frac{\beta_{3} \delta \omega}{\left(\omega \beta_{1}+\gamma \beta_{2}+k \gamma \beta_{2}\right)^{*}}
\end{aligned}
$$

Dari persamaan (17), (18) dan (19) diperoleh

Matriks Jacobian pada titik ekuilibrium bebas penyakit $P_{0}\left(0,0,0, \frac{A}{\mu}\right)$ adalah

If $\left(P_{0}\right)=$

$\left[\begin{array}{cccc}\frac{\beta_{1} A}{\mu}-\delta & \frac{\beta_{z} A}{\mu} & \frac{\beta_{3} A}{\mu}-n & 0 \\ \gamma & -\omega & 0 & 0 \\ 0 & k & -1 & 0 \\ -\alpha_{1} & -\alpha_{2} & 0 & -1\end{array}\right]$

Polinomial karakteristik dari $I f\left(P_{0}\right)$ adalah $P(\lambda)=\operatorname{det}\left(\lambda I-J f\left(P_{0}\right)\right)$,

dengan $I$ adalah matriks identitas ukuran $4 \times 4$.

Persamaan karakteristiknya adalah $\operatorname{det}\left(\lambda I-J f\left(P_{0}\right)\right)$

$\Leftrightarrow \operatorname{det}\left[\begin{array}{cccc}\lambda-\frac{\beta_{1} A}{\mu}+\delta & -\frac{\beta_{2} A}{\mu} & -\frac{\beta_{3} A}{\mu} & 0 \\ -\gamma & \lambda+\omega & 0 & 0 \\ 0 & -k & \lambda+1 & 0 \\ a_{1} & a_{2} & 0 & \lambda+1\end{array}\right]$

$\Leftrightarrow(\lambda+1)\left|\begin{array}{ccc}\lambda-\frac{\beta_{1} A}{\mu}+\delta & -\frac{\beta_{2} A}{\mu} & -\frac{\beta_{g} A}{\mu} \\ -\gamma & \lambda+\omega & 0 \\ 0 & -k & \lambda+1\end{array}\right|$

$\Leftrightarrow(\lambda+1)\left[\left(\lambda-\frac{\beta_{1} A}{\mu}+\delta\right)(\lambda+\omega)(\lambda+\right.$

1) $\left.+\left(-\frac{\beta_{g} A}{\mu}\right)(\gamma k)-\left(\frac{\gamma \beta_{x} A}{\mu}\right)(\lambda+1)\right]$ 


$$
\begin{aligned}
& \Leftrightarrow(\lambda+1)\left[( \lambda - \frac { \beta _ { 1 } A } { \mu } + \delta ) \left(\lambda^{2}+\lambda+\omega \lambda+\right.\right. \\
& \left.\omega)-\frac{\beta_{\Omega} A \gamma k}{\mu}-\frac{\gamma \beta_{2} A \lambda}{\mu}-\frac{\gamma \beta_{2} A}{\mu}\right] \\
& \Leftrightarrow(\lambda+1)\left[\lambda^{3}-\frac{\beta_{1} A \lambda^{2}}{\mu}+\delta \lambda^{2}+\lambda^{2}-\frac{\beta_{1} A \lambda}{\mu}+\right. \\
& \delta \lambda+\lambda^{2} \omega-\frac{\beta_{1} A \omega \lambda}{\mu}+\delta \omega \lambda+\lambda \omega- \\
& \left.\quad \frac{\beta_{1} A \omega}{\mu}+\delta \omega-\frac{\beta_{2} A \gamma k}{\mu}-\frac{\gamma \beta_{2} A \lambda}{\mu}-\frac{\gamma \beta_{2} A}{\mu}\right] \\
& \Leftrightarrow(\lambda+1)\left[\lambda^{3}+\left(\delta+\omega+1-\frac{\beta_{1} A}{\mu}\right) \lambda^{2}+\right. \\
& \left(\delta+\delta \omega+\omega-\frac{\beta_{1} A}{\mu}-\frac{\beta_{1} A \omega}{\mu}-\frac{\gamma \beta_{2} A}{\mu}\right) \lambda+\delta \omega- \\
& \left.\frac{\beta_{1} A \omega}{\mu}-\frac{\beta_{g} A \gamma k}{\mu}-\frac{\gamma \beta_{2} A}{\mu}\right] .
\end{aligned}
$$

Persamaan (20) dapat ditulis menjadi $(\lambda+1)\left(\lambda^{3}+A \lambda^{2}+B \lambda+C\right)=0$

$$
\text { dengan }
$$

$$
\begin{aligned}
& A=\delta+\omega+1-\frac{\beta_{1} A}{\mu}, \\
& B=\delta+\delta \omega+\omega-\frac{\beta_{1} A}{\mu}-\frac{\beta_{1} A \omega}{\mu}-\frac{\gamma \beta_{2} A}{\mu}, \\
& C=\delta \omega-\frac{\beta_{1} A \omega}{\mu}-\frac{\beta_{2} A Y}{\mu}-\frac{\beta_{g} A k y}{\mu} .
\end{aligned}
$$$$
\text { Karena } R_{0}=\frac{A\left(\beta_{1} \omega+\beta_{2} \gamma+\beta_{g} k \gamma\right)}{\delta \omega \mu}>1 \text {, maka }
$$$$
A\left(\beta_{1} \omega+\beta_{2} \gamma+\beta_{3} k \gamma\right)>\delta \omega \mu
$$$$
\Leftrightarrow \beta_{1} A \omega+\beta_{2} A \gamma+\beta_{3} A k \gamma>\delta \omega \mu
$$$$
\Leftrightarrow \frac{\beta_{1} A \omega}{\mu}+\frac{\beta_{2} A Y}{\mu}+\frac{\beta_{\mathrm{g}} A k \gamma}{\mu}>\delta \omega
$$$$
\Leftrightarrow \delta \omega-\frac{\beta_{1} A \omega}{\mu}-\frac{\beta_{2} A Y}{\mu}-\frac{\beta_{\Omega} A k \gamma}{\mu}<0=C
$$

Berdasarkan kriteria Routh-Hurwitz maka titik ekuilibrium $P_{0}\left(0,0,0, \frac{A}{\mu}\right)$ tidak stabil.

b. Matriks Jacobian pada titik ekuilibrium endemik $P^{*}=\left(E^{*}, I^{*}, R^{*}, N^{*}\right)$ adalah $/\left(P^{*}\right)$.

Jika $\quad m=\frac{\delta \omega}{\left(\omega \beta_{1}+\gamma \beta_{2}+k \gamma \beta_{g}\right)} \quad, \quad$ maka polinomial karakteristik dari $I f\left(P^{*}\right)$ adalah

$$
P(\lambda)=\operatorname{det}\left(\lambda I-J f\left(P^{*}\right)\right),
$$

dengan $I$ adalah matriks identitas ukuran $4 \times 4$.

Persamaan karakteristiknya adalah

$$
\begin{aligned}
& \operatorname{det}\left(\lambda I-J f\left(P^{*}\right)\right)=0 \\
& \beta \operatorname{det}\left[\begin{array}{cccc}
\lambda-m \beta_{1}+n+\delta & -m \beta_{2}+n & -m \beta_{2}+n & -n \\
-\eta & \lambda+\omega & 0 & 0 \\
0 & -k & \lambda+1 & 0 \\
a_{1} & a_{2} & 0 & \lambda+1
\end{array}\right]=0 \\
& \theta n\left[\left(a_{1}\right)(a+1)(a+\omega)-\left(a_{2}\right)(a+1)(-\gamma)\right] \\
& +(\lambda+1)\left[\left(\lambda-m \beta_{1}+n+\delta\right)(\lambda+\omega)(\lambda+1)+\left(-m \beta_{\beta}+n\right)(-\gamma)(-k)\right. \\
& \left.-(\lambda+1)(-\gamma)\left(-m \beta_{2}+n\right)\right]=0 \\
& a(a+1)\left[a_{1} n(a+\omega)+p m a_{2}+(a+1)\left(a-m \beta_{1}+n+\delta\right)(a+\omega)\right. \\
& \left.-(\lambda+1)(-\gamma)\left(-m \beta_{2}+n\right)+k y\left(n-m \beta_{8}\right)\right]=0 \\
& \theta(\lambda+1)\left[a_{1} n+a_{1} n \omega+p n a_{2}+\left(\lambda-m \beta_{1}+n+\delta\right)\left(\lambda^{2}+\lambda+\lambda \omega+\omega\right)\right. \\
& \left.+(n p+\gamma)\left(-m \beta_{2}+n\right)+k \gamma\left(n-m \beta_{2}\right)\right]=0
\end{aligned}
$$

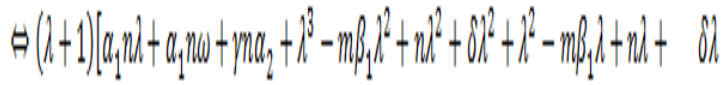

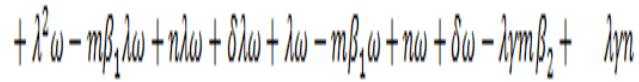

$$
\begin{aligned}
& \left.-m m_{2}+m+(w)\left(n-m b_{3}\right)\right]=0
\end{aligned}
$$

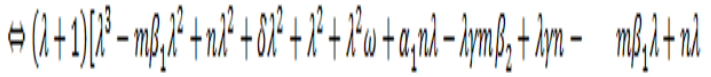

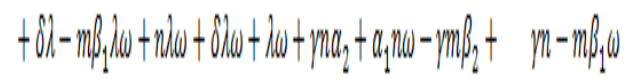
$\left.+m(\omega)+6(w+k)\left(n-m \beta_{3}\right)\right]=0$

$$
\begin{aligned}
& \Leftrightarrow(\lambda+1)\left[\lambda^{3}+\left(-m \beta_{1}+n+\delta+1+\right.\right. \\
& \omega) \lambda^{2}+\left(\alpha_{1} n-\gamma m \beta_{2}+\gamma n-m \beta_{1}+n+\right. \\
& \left.\delta-m \beta_{1} \omega+n \omega+\delta \omega+\omega\right) \lambda+\gamma n \alpha_{2}+ \\
& \alpha_{1} n \omega-\gamma m \beta_{2}+\gamma n-m \beta_{1} \omega+n \omega+ \\
& \left.\delta \omega+k \gamma\left(n-m \beta_{3}\right)\right]=0 \\
& \Leftrightarrow(\lambda+1)\left[\lambda^{3}+\left(1+\omega+n-m \beta_{1}+\delta\right) \lambda^{2}+\right. \\
& \left(\omega+n \alpha_{1}+\left(n-m \beta_{1}+\delta\right)(1+\omega)+\right. \\
& \left.\gamma\left(n-m \beta_{2}\right)\right) \lambda+n\left(\alpha_{2} \gamma+\alpha_{1} \omega\right)+ \\
& \gamma\left(n-m \beta_{2}\right)+k \gamma\left(n-m \beta_{3}\right)+ \\
& \left.\quad \omega\left(n-m \beta_{1}+\delta\right)\right]=0 .
\end{aligned}
$$

Persamaan (22) dapat ditulis menjadi $(\lambda+1)\left(\lambda^{3}+A \lambda^{2}+B \lambda+C\right)=0$

dengan

$$
A=1+\omega+n-m \beta_{1}+\delta_{,}
$$




$$
\begin{aligned}
& B=\omega+n \alpha_{1}+\left(n-m \beta_{1}+\delta\right)(1+\omega)+ \\
& \gamma\left(n-m \beta_{2}\right), \\
& C=n\left(\alpha_{2} \gamma+\alpha_{1} \omega\right)+\gamma\left(n-m \beta_{2}\right)+ \\
& k \gamma\left(n-m \beta_{3}\right)+\omega\left(n-m \beta_{1}+\delta\right)
\end{aligned}
$$

Salah satu nilai eigen dari persamaan (23) adalah -1 , nilai - nilai eigen yang lain merupakan akar - akar dari polinomial berikut

$$
Q(\lambda)=\lambda^{3}+A \lambda^{2}+B \lambda+C
$$

Perhatikan bahwa

$$
\begin{aligned}
& \quad A=1+\omega+n-m \beta_{1}+\delta \\
& =1+\omega+n-\frac{\delta \omega \beta_{1}}{\left(\omega \beta_{1}+\gamma \beta_{2}+k \gamma \beta_{g}\right)}+ \\
& \frac{\delta\left(\omega \beta_{1}+\gamma \beta_{2}+k \gamma \beta_{\mathrm{g}}\right)}{\left(\omega \beta_{1}+\gamma \beta_{2}+k \gamma \beta_{\mathrm{g}}\right)} \\
& =1+\omega+n+\frac{\delta\left(\omega \beta_{1}+\gamma \beta_{2}+k \gamma \beta_{\mathrm{g}}\right)-\delta \omega \beta_{1}}{\omega \beta_{1}+\gamma \beta_{2}+k \gamma \beta_{\mathrm{g}}} \\
& =1+\omega+n+\frac{\delta\left(\omega \beta_{1}+\gamma \beta_{2}+k \gamma \beta_{\mathrm{g}}-\delta \omega \beta_{1}\right)}{\omega \beta_{1}+\gamma \beta_{2}+k \gamma \beta_{\mathrm{g}}} \\
& =1+\omega+n+\frac{\delta\left(\gamma \beta_{2}+k \gamma \beta_{\mathrm{g}}\right)}{\omega \beta_{1}+\gamma \beta_{2}+k \gamma \beta_{\mathrm{g}}}>0 .
\end{aligned}
$$

Selanjutnya

$$
\begin{aligned}
& B=\omega+n \alpha_{1}+\left(n-m \beta_{1}+\delta\right)(1+\omega)+ \\
& \gamma\left(n-m \beta_{2}\right) \\
& =\omega+n \alpha_{1}+n-m \beta_{1}+\delta+n \omega- \\
& m \beta_{1} \omega+\delta \omega+\gamma n-\gamma m \beta_{2} \\
& =\omega+n\left(\alpha_{1}+1+\omega+\gamma\right)+\delta+\delta \omega-m\left(\beta_{1}+\beta_{1} \omega+\gamma \beta_{2}\right) \\
& = \\
& \omega+n(\omega+\delta)+\delta+\delta \omega- \\
& \frac{\delta \omega}{\left.\omega \beta_{1}+\gamma \beta_{2}+k \gamma \beta_{g}\right)}\left(\beta_{1}+\beta_{1} \omega+\gamma \beta_{2}\right) \\
& =\quad \\
& \begin{array}{l}
\omega+n(\omega+\delta)+ \\
\frac{(\delta+\delta \omega)\left(\omega \beta_{1}+\gamma \beta_{2}+k \gamma \beta_{g}\right)-\delta \omega\left(\beta_{1}+\beta_{1} \omega+\gamma \beta_{2}\right)}{\omega \beta_{1}+\gamma \beta_{2}+k \gamma \beta_{g}}
\end{array}
\end{aligned}
$$

$$
\begin{aligned}
& = \\
& \omega+n(\omega+\delta)+ \\
& \frac{\operatorname{s}\left(\omega \beta_{1}+\gamma \beta_{2}+k \gamma\left(\beta_{3}\right)+\operatorname{d\omega }\left(\omega \beta_{1}+\gamma \beta_{2}+k \gamma\left(\beta_{3}\right)-\operatorname{d\omega }\left(\beta_{1}+\beta_{1} \omega+\gamma \beta_{2}\right)\right.\right.}{\omega} \\
& \omega \beta_{1}+\gamma \beta_{2}+k \gamma \beta_{3} \\
& = \\
& \omega+n(\omega+\delta)+ \\
& \underline{\delta \omega \beta_{1}+\delta \gamma \beta_{2}+\delta k \gamma \beta_{8}+\delta \omega^{2} \beta_{1}+\delta \omega \gamma \beta_{2}+\delta \omega k \gamma \beta_{8}-\delta \omega \beta_{1}-\delta \omega^{2} \beta_{1}-\delta \omega \gamma \beta_{2}} \\
& \omega \beta_{1}+\gamma \beta_{2}+k \gamma \beta_{8} \\
& =\omega+n(\omega+\delta)+\frac{\delta y \beta_{2}+\delta k \gamma \beta_{\mathrm{g}}+\delta \omega k \gamma \beta_{\mathrm{g}}}{\omega \beta_{1}+\gamma \beta_{\mathrm{z}}+k \gamma \beta_{\mathrm{g}}} \\
& =\omega+n(\omega+\delta)+\frac{\delta \gamma\left(\beta_{2}+k \beta_{3}+\omega k \beta_{3}\right)}{\omega \beta_{1}+\gamma \beta_{2}+k \gamma \beta_{3}} \\
& =\omega+n(\delta+\omega)+\frac{\delta y\left[\beta_{2}+k(1+\omega) \beta_{s}\right]}{\omega \beta_{1}+\gamma \beta_{2}+k y \beta_{g}}>0 .
\end{aligned}
$$

Selanjutnya

$C=n\left(\alpha_{2} \gamma+\alpha_{1} \omega\right)+\gamma\left(n-m \beta_{2}\right)+k \gamma\left(n-m \beta_{3}\right)+\omega\left(n-m \beta_{1}+\delta\right)$ $=n a_{2} \gamma+n a_{1} \omega+\gamma n-\gamma m \beta_{2}+k \gamma n-k \gamma m \beta_{3}+\omega n-\omega m \beta_{1}+\omega \delta$ $=\left(n \alpha_{2}+n-m \beta_{2}+k n-k m \beta_{3}\right) \gamma+\left(n \alpha_{1}+n-m \beta_{1}+\delta\right) \omega$ $=\left(\left(1+\alpha_{2}+k\right) n-m \beta_{2}-k m \beta_{3}\right) y+\left(\left(1+a_{1}\right) n-m \beta_{1}+\delta\right) \omega$ $=\left(\omega n-m \beta_{2}-k m \beta_{3}\right) \gamma+\left((\delta-\gamma) n-m \beta_{1}+\delta\right) \omega$ $=\omega n \gamma-m \beta_{2} \gamma-k m \beta_{3} \gamma+\left(\delta n-\gamma n-m \beta_{1}+\delta\right) \omega$ $=\omega n \gamma-m \beta_{2} \gamma-k m \beta_{3} \gamma+\delta n \omega-\gamma n \omega-m \beta_{1} \omega+\delta \omega$ $=\left(-\gamma \beta_{2}-k \gamma \beta_{3}-\omega \beta_{1}\right) m+\delta n \omega+\delta \omega$ $=-\left(\omega \beta_{1}+\gamma \beta_{2}+k \gamma \beta_{3}\right) \frac{\delta \omega}{\left(\omega \beta_{1}+\gamma \beta_{2}+k \gamma \beta_{3}\right)}+\delta n \omega+\delta \omega$ $=-\delta \omega+\delta n \omega+\delta \omega$

$$
=\delta n \omega>0 \text {. }
$$

Selanjutnya

$A B-C=$

$\left(1+\omega+n+\frac{\delta\left(y \beta_{2}+k \gamma \beta_{g}\right)}{\omega \beta_{1}+\gamma \beta_{2}+k \gamma \beta_{g}}\right)(\omega+$

$\left.n(\delta+\omega)+\frac{\delta y\left[\beta_{2}+k(1+\omega) \beta_{g}\right]}{\omega \beta_{1}+\gamma \beta_{2}+k \gamma \beta_{g}}\right)-\delta n \omega$

$$
>(1+\omega+n)(\omega+n \delta+n \omega)-\delta n \omega
$$

$=\omega+n \delta+n \omega+\omega^{2}+n \delta \omega+n \omega^{2}+\omega n+n^{2} \delta+n^{2} \omega-\delta n \omega$ $=(1+n) \omega^{2}+\left(n^{2}+2 n+1\right) \omega+n \delta(1+$ $A B-C>0$.

Dari Persamaan (25), (26), (27) dan (28) diperoleh bahwa

$$
A>0, B>0, C>0 \text { dan } A B>C \text {. }
$$


Berdasarkan nilai koefisien polinomial $\mathrm{Q}(\lambda)$ pada Persamaan (24) diperoleh

$a_{0}=1, a_{1}=A, a_{2}=B$

$$
a_{3}=C_{3} a_{4}=0, a_{5}=0 .
$$

Dari nilai diatas, dapat disusun matriks Hurwitz sebagai berikut

$$
H=\left[\begin{array}{lll}
a_{1} & a_{0} & 0 \\
a_{3} & a_{2} & a_{1} \\
a_{5} & a_{4} & a_{3}
\end{array}\right]=\left[\begin{array}{ccc}
A & 1 & 0 \\
C & B & A \\
0 & 0 & C
\end{array}\right] .
$$

Berdasarkan matriks Hurwitz diatas dapat ditentukan determinan Hurwitz yaitu

$\Delta_{1}=a_{1}=A$

$\Delta_{2}=\left|\begin{array}{ll}a_{1} & a_{0} \\ a_{3} & a_{2}\end{array}\right|=\left|\begin{array}{ll}A & 1 \\ C & B\end{array}\right|=A B-C$

$\Delta_{3}=\left|\begin{array}{lll}a_{1} & a_{0} & 0 \\ a_{3} & a_{2} & a_{1} \\ a_{5} & a_{4} & a_{3}\end{array}\right|=\left|\begin{array}{ccc}A & 1 & 0 \\ C & B & A \\ 0 & 0 & C\end{array}\right|=$

$A B C-C^{2}=(A B-C) C$.

Berdasarkan nilai $A, B$ dan $C$, maka dapat ditentukan bahwa

1. Karena nilai $A>0$ maka $\Delta_{1}=A>0$

2. Karena

$A>0, B>0, C>0$

nilai

$A B>C$, maka

$\Delta_{2}=A B-C>0$

3. Karena

nilai

$A>0, B>0, C>0 \quad$ dan

$A B>C$, maka

$\Delta_{3}=(A B-C) C>0$.

Karena nilai $\Delta_{1}>0, \Delta_{2}>0$ dan $\Delta_{3}>0$ maka polinomial $\mathrm{Q}(\lambda)$ mempunyai pembuat nol yang bagian realnya negatif. Hal ini berakibat semua bagian real nilai eigen pada matriks $I f\left(P^{*}\right)$ bernilai negatif untuk $R_{0}>1$. Jadi titik ekuilibrium $P^{*}=\left(E^{*}{ }_{3}{ }^{*}, R^{*}, N^{*}\right)$ stabil asimtotik lokal.

Selanjutnya akan diselidiki kestabilan global titik ekuilibrium endemik pada Sistem berikut :

$$
\begin{aligned}
& \frac{d S}{d \tau}=\frac{A}{\mu}-\beta_{1} S E-\beta_{2} S I-\beta_{3} S R-S \\
& \frac{d E}{d \tau}=\beta_{1} S E+\beta_{2} S I+\beta_{3} S R-\delta E
\end{aligned}
$$

$\frac{d I}{d \tau}=\gamma E-\omega I$

$\frac{d R}{d \tau}=k I-R$

$\frac{d N}{d t}=\frac{A}{\mu}-N$

jika $\alpha_{1}=\alpha_{2}=0$.

\section{Teorema 2}

Perhatikan sistem berikut

$x^{\prime}=f(x), x \in T$.

Jika syarat - syarat berikut dipenuhi

(1) Sistem (34) ada untuk suatu himpunan compact absorbing $K \subset T$ dan mempunyai suatu titik ekuilibrium tunggal $P$ di $T$;

(2) $P$ stabil asimtotik lokal;

(3) Sistem (34) memenuhi kriteria Poincare - Bendixson;

(4) Suatu orbit periodik pada Sistem (34) adalah stabil asimtotik orbital,

maka hanya titik ekuilibrium $P$ yang stabil asimtotik global di $T$.

Bukti :

Cukup dibuktikan bahwa $\bar{x}$ menarik semua titik di $T$. Misalkan $U$ basin of attraction dari $\bar{x}$, yaitu himpunan dari semua $x_{0}$ sedemikian sehingga $x\left(t, x_{0}\right)$ konvergen ke $\bar{x}$. Jadi $U$ merupakan himpunan terbuka tak kosong yang stabil asimtotik pada $\bar{x}$. Teorema akan terbukti dengan menunjukkan $\quad T \subset U$. Pembuktian dilakukan dengan kontradiksi, maka batas $\partial U$ pada $U$ mempunyai irisan tak kosong $I$ dengan $T$. Karena $U$ dan $\bar{U}$ invarian dan $U$ terbuka, $\partial U=U-\bar{U}$ juga invariant, maka $I$ merupakan invarian positif. Oleh karena itu $I$ memuat suatu himpunan omega limit kompak $\Omega$ yang tak kosong. Karena sistem (18) ada untuk suatu himpunan compact absorbing $K \subset T$, maka haruslah 
$\Omega \cap \partial T=\emptyset$. Karena $\Omega$ tidak memuat titik ekuilibrium, berdasarkan sifat PoincareBendixson maka $\Omega$ merupakan orbit tertutup, dan berdasarkan asumsi (4) maka $\Omega$ stabil asimtotik orbit. Ini kontradiksi, karena $\Omega$ anggota himpunan $\alpha$ - limit pada suatu orbit dalam $U$.

Titik ekuilibrium Sistem (29) s.d (33) diperoleh dengan menjadikan ruas kanan masing - masing persamaan sama dengan nol, yaitu :

$$
\begin{aligned}
& \frac{A}{\mu}-\beta_{1} S E-\beta_{2} S I-\beta_{3} S R-S=0 \\
& \beta_{1} S E+\beta_{2} S I+\beta_{3} S R-\delta E=0 \\
& \gamma E-\omega I=0 \\
& k I-R=0 \\
& \frac{A}{\mu}-N=0 .
\end{aligned}
$$

Dari Persamaan (37) diperoleh $\gamma E-\omega I=0 \Leftrightarrow \gamma E=\omega I$

$$
\Leftrightarrow E=\frac{\omega I}{Y} .
$$

Dari Persamaan (40) diperoleh $k I-R=0 \Leftrightarrow R=k I$.

Jika Persamaan (40) dan (41) disubstitusi ke dalam Persamaan (36) diperoleh $\beta_{1} S E+\beta_{2} S I+\beta_{3} S R-\delta E=0$ $\Leftrightarrow \frac{\beta_{1} S \omega I}{\gamma}+\beta_{2} S I+\beta_{3} S k I-\frac{\delta \omega I}{\gamma}=0$ $\Leftrightarrow\left(\frac{\beta_{1} S \omega}{\gamma}+\beta_{2} S+\beta_{3} S k-\frac{\delta \omega}{\gamma}\right) I=0$ $\Leftrightarrow I=0$ atau $\frac{\beta_{1} S \omega}{\gamma}+\beta_{2} S+\beta_{3} S k=\frac{\delta \omega}{\gamma}$.

Jika $I>0$ maka

$$
\begin{aligned}
& \frac{\beta_{1} S \omega}{\gamma}+\beta_{2} S+\beta_{3} S k=\frac{\delta \omega}{\gamma} \\
& \Leftrightarrow\left(\frac{\beta_{1} \omega}{\gamma}+\beta_{2}+\beta_{3} k\right) S=\frac{\delta \omega}{\gamma}
\end{aligned}
$$

$$
\begin{aligned}
& \Leftrightarrow\left(\frac{\beta_{1} \omega+\beta_{2} \gamma+\beta_{3} k \gamma}{\gamma}\right) S=\frac{\delta \omega}{\gamma} \\
& \Leftrightarrow S=\frac{\delta \omega}{\beta_{1} \omega+\beta_{2} \gamma+\beta_{3} k \gamma} .
\end{aligned}
$$

Perhatikan bahwa

$$
S+E+I+R=N
$$

$\Leftrightarrow S+\frac{\omega I}{\gamma}+I+k I=\frac{A}{\mu}$

$\Leftrightarrow\left(\frac{\omega}{\gamma}+1+k\right) I=\frac{A}{\mu}-S$

$\Leftrightarrow\left(\frac{\omega}{y}+1+k\right) I=\frac{A}{\mu}-\frac{\delta \omega}{\beta_{1} \omega+\beta_{2} \gamma+\beta_{2} k \gamma}$

$\Leftrightarrow\left(\frac{\omega+\gamma+k \gamma}{\gamma}\right) I=\frac{A\left(\beta_{1} \omega+\beta_{2} \gamma+\beta_{3} k \gamma\right)-\delta \omega \mu}{\mu\left(\beta_{1} \omega+\beta_{2} \gamma+\beta_{3} k \gamma\right)}$

$\Leftrightarrow I=\frac{A \gamma\left(\beta_{1} \omega+\beta_{2} \gamma+\beta_{2} k \gamma\right)-\delta \gamma \omega \mu}{\mu(\omega+\gamma+k \gamma)\left(\beta_{1} \omega+\beta_{2} \gamma+\beta_{2} k \gamma\right)}$.

Dari Persamaan (41) diperoleh $R=k I$

$=\frac{k A \gamma\left(\beta_{1} \omega+\beta_{2} \gamma+\beta_{3} k \gamma\right)-k \delta \gamma \omega \mu}{\mu(\omega+\gamma+k \gamma)\left(\beta_{1} \omega+\beta_{2} \gamma+\beta_{3} k \gamma\right)}$.

Dari persamaan (40) diperoleh

$E=\frac{\omega I}{\gamma}$

$=\frac{\omega A \gamma\left(\beta_{1} \omega+\beta_{2} \gamma+\beta_{3} k \gamma\right)-\delta \gamma \mu \omega^{2}}{\mu \gamma(\omega+\gamma+k \gamma)\left(\beta_{1} \omega+\beta_{2} \gamma+\beta_{3} k \gamma\right)}$.

Jadi titik ekuilibrium endemik Sistem (29) s.d (33) jika $\alpha_{1}=\alpha_{2}=0$ adalah $S=\frac{\delta \omega}{\beta_{1} \omega+\beta_{2} \gamma+\beta_{3} k \gamma}$

$E=\frac{\omega A \gamma\left(\beta_{1} \omega+\beta_{2} \gamma+\beta_{2} k \gamma\right)-\delta \gamma \mu \omega^{2}}{\mu \gamma(\omega+\gamma+k \gamma)\left(\beta_{1} \omega+\beta_{2} \gamma+\beta_{2} k \gamma\right)}$

$I=\frac{A \gamma\left(\beta_{1} \omega+\beta_{2} \gamma+\beta_{3} k \gamma\right)-\delta \gamma \omega \mu}{\mu(\omega+\gamma+k \gamma)\left(\beta_{1} \omega+\beta_{2} \gamma+\beta_{3} k \gamma\right)}$

$R=\frac{k A \gamma\left(\beta_{1} \omega+\beta_{2} \gamma+\beta_{3} k \gamma\right)-k \delta \gamma \omega \mu}{\mu(\omega+\gamma+k \gamma)\left(\beta_{1} \omega+\beta_{2} \gamma+\beta_{3} k \gamma\right)}$.

\section{Teorema 3}

Jika $R_{0}>1$ dan $\alpha_{1}=\alpha_{2}=0$, maka Sistem (5) s.d (8) adalah kooperatif.

\section{Bukti}

Misalkan $S=x_{1}, E=x_{2}, I=x_{3}$ dan $R=x_{4}$ maka Sistem (5) s.d (8) dapat dinyatakan dengan 


$$
\begin{gathered}
x_{1}^{\prime}=\frac{A}{\mu}-\beta_{1} S\left(\frac{A}{\mu}-x_{1}-x_{3}-x_{4}\right)- \\
\beta_{2} S\left(\frac{A}{\mu}-x_{1}-x_{2}-x_{4}\right)-\beta_{3} S\left(\frac{A}{\mu}-x_{1}-\right. \\
\left.x_{2}-x_{3}\right)-x_{1} \\
x_{2}^{\prime}=\beta_{1} S x_{2}+\beta_{2} S x_{3}+\beta_{3} S x_{4}-\delta x_{2}, \\
x_{3}^{\prime}=\gamma x_{2}-\omega x_{3} \\
x_{4}^{\prime}=k x_{3}-x_{4}
\end{gathered}
$$

Selanjutnya, sistem ini dapat dinyatakan dalam bentuk

$\left(\begin{array}{l}x_{1}^{\prime} \\ x_{2}^{\prime} \\ x_{2}^{\prime} \\ x_{4}^{\prime}\end{array}\right)=\left[\left(\begin{array}{cccc}\left(\beta_{1}+\beta_{2}+\beta_{2}\right) S & \left(\beta_{2}+\beta_{2}\right) S & \left(\beta_{1}+\beta_{2}\right) S & \left(\beta_{1}+\beta_{2}\right) S \\ 0 & \beta_{2} S-\gamma & \beta_{2} S & \beta_{2} S \\ 0 & \gamma & -k & 0 \\ 0 & 0 & k & 0\end{array}\right)-\left(\begin{array}{llll}1 & 0 & 0 & 0 \\ 0 & 1 & 0 & 0 \\ 0 & 0 & 1 & 0 \\ 0 & 0 & 0 & 1\end{array}\right)\left(\begin{array}{l}x_{1} \\ x_{2} \\ x_{2} \\ x_{4}\end{array}\right)+\left(\begin{array}{l}C_{1} \\ C_{2} \\ C_{2} \\ C_{4}\end{array}\right)\right.$,

atau dapat ditulis dalam bentuk

$$
x^{\prime}=(A(t)-I) x+C(t),
$$

Dengan $\quad x=\left(x_{1}, x_{2}, x_{3}, x_{4}\right) \in \mathbb{R}^{4}, \quad I$ menyatakan matriks identitas pada matriks $4 \times 4, C(t)$ adalah fungsi vektor, dan

$A=\left(\begin{array}{cccc}\left(\beta_{1}+\beta_{2}+\beta_{2}\right) S & \left(\beta_{2}+\beta_{2}\right) S & \left(\beta_{2}+\beta_{2}\right) S & \left(\beta_{1}+\beta_{2}\right) S \\ 0 & \beta_{2} S-\gamma & \beta_{2} S & \beta_{2} S \\ 0 & \gamma & -k & 0 \\ 0 & 0 & k & 0\end{array}\right)$

Elemen - elemen dari off-diagonal matriks ini non-negative, sistem secara keseluruhan quasimonotone, maka Sistem (5) s.d (8) kooperatif berkaitan dengan partial ordering yang didefinisikan pada orthant $K=\left\{(S, E, I, R) \in \mathbb{R}^{4}: S \geq 0, E \geq 0, I \geq\right.$ $0, R \geq 0\}$

\section{SIMPULAN}

Adapun kesimpulan dari penelitian ini adalah

1.Model matematika epidemi SEIR dengan adanya kemampuan infeksi pada periode laten, infeksi dan sembuh adalah :

$$
\begin{aligned}
& \frac{d S}{d t}=A-\beta_{10} S E-\beta_{20} S I-\beta_{30} S R-\mu S \\
& \frac{d E}{d t}=\beta_{10} S E+\beta_{20} S I+\beta_{30} S R-\gamma_{0} E- \\
& \left(\mu+\alpha_{10}\right) E \\
& \frac{d I}{d t}=\gamma_{0} E-k_{0} I-\left(\mu+\alpha_{20}\right) I \\
& \frac{d R}{d t}=k_{0} I-\mu R .
\end{aligned}
$$

2.Titik - titik ekuilibrium model tersebut adalah : Jika $R_{0}>1$ maka model (1) mempunyai dua titik ekuilibrium yaitu titik ekuilibrium bebas penyakit $P_{0}\left(\frac{A}{\mu}, 0,0,0\right)$ dan titik ekuilibrium endemik tunggal yaitu $P^{*}\left(S^{*}, E^{*}{ }_{,}^{*} I_{,}, R^{*}\right)$, dengan

$S=\frac{A\left(\alpha_{1} \omega+\alpha_{2} \gamma\right)}{\left(\beta_{1} \omega+\beta_{2} \gamma+\beta_{\mathrm{g}} k \gamma\right)(A-\mu N)+\mu\left(\alpha_{1} \omega+\alpha_{2} \gamma\right)}$

$E=\frac{\omega[A-\mu N]}{\mu\left(\omega \alpha_{1}+\gamma \alpha_{2}\right)}$

$I=\frac{\gamma[A-\mu N]}{\omega\left(\mu \alpha_{1}\right)+\gamma \mu \alpha_{2}}$

$R=\frac{k \gamma[A-\mu N]}{\mu\left(\alpha_{1} \omega+\alpha_{2} \gamma\right)}$

3.Kestabilan titik - titik ekuilibrium model tersebut adalah : Jika $R_{0}>1$ maka titik ekuilibrium endemik $P^{*}\left(S^{*}, E^{*}, I^{*}, R^{*}\right)$ stabil asimtotik global. Hal ini menunjukkan bahwa dalam jangka waktu yang panjang individu yang ada di sekitar titik ekuilibrium $P^{*}\left(S^{*}, E^{*}, I^{*}, R^{*}\right)$ masih terdapat individu yang terjangkit penyakit atau tetap terjadi penyebaran penyakit.

\section{SARAN}

Karena berbagai keterbatasan, penulis menyadari penelitian dan tulisan ini masih banyak kekurangannya. Banyak hal yang belum tercakup dalam penelitan ini. Perlu dikaji lebih lanjut generalisasi untuk kestabilan model epidemi lainnya dan simulasi numeriknya.

\section{DAFTAR PUSTAKA}

Anton H, dan Rorres,C.,2004. Aljabar Linear Elementer Versi Aplikasi, Edisi Kedelapan, alih bahasa oleh Indriasari, R dan Harmaen,I., Erlangga, Jakarta.

Arrowsmith, D.R. dan Place, C.M., 1992, Dynamical System Differential Equation, Maps and Chaotic Behaviour, Chapman \& Hall Mathematic, London.

Bazaraa, M.S., Sheraly, H.D, and Shetty, C.M., Nonlinear Programming Theory and Algorithms, John Wiley \& Sons, Inc, New York, 1993. 
Becerra, M.V., 2008. La Salle's Invariant Set Theory, http://www.personal.rdg.ac.uk/ shs 99vmb/notes/anc/lecture3.pdf

Boyd, Stephen, 2008, Basic Lyapunov Theory, Stanford University, http://www.stanford.edu/class/ee363 lyap.pdf

Capazzo, V., Mathematical Structures of Epidemic Systems, Springer-Verlag, Heidelberg, 2008.

Chong, K.P and Stainslow, H.Z., An Introduction to Optimization, John Wiley \& Sons, University of New Hampshire, 1984.

Gantmacher, F.R., 1959, The Theory of Matrices, Chelsea Publishing Company, New York.

Hanh, Wolfgang, 1967, Stability of Motion, Springer - Verlag, New York.

Hirsch, M.W., dan Smith, Monotone Dynamical Systems, University of California, Berkeley, 2004.

Iwami. S, Takeuchi, Y., dan Liu, X., 2007. Avian - human Influenza Epidemic Model, Mathematical Biosciences 2007, hal 1-25.

Feng J., dan Hadeler,K.P., Qualitative Behaviour of Some Simple Networks, Mathematical Gen. 1996, hal 5019-5033.

Kocak, H. dan Hole, J.K., 1991, Dynamic and Bifurcation, Springer - Verlag, New York.

Leon, J.S., Aljabar Linear dan Aplikasinya, Edisi Kelima, Alih bahasa olehBondan,A. Erlangga, Jakarta, 1998.

Luenberger, G.D., 1979, Introduction to Dynamic System Theory, Models \& Aplication, John Wiley \& Sons, New York.

Olsder, G.J., 1994, Mathematical System Theory, Delftse Uitgevers Maatschappij, Netherlands.
Perko L., 1991, Differential Equations and Dynamical Systems, Springer Verlag, New York.

Ross, S.L., Differential Equations, $3^{\text {rd }}$ edition, John Wiley \& Sons, University of New Hampshire, 1984.

Verhultz, Ferdinand, 1990, Nonlinear Differential Equations and Dynamical Systems, Springer Verlag, Berlin.

Wiggins S, 1990, Introduction to Applied Nonlinear Dynamical Systems and Chaos, Springer - Verlag, New York. 